- the texts have to include detailed instructions about how to engage with the various course components, including the texts themselves

- the texts have to provide pacing

- the sequence of topics has to be prepared with great care, and it is essential to specify clearly and completely the knowledge and skills that students are expected to bring to the course

- the texts have to be "student-active", for example by providing in-text questions that enable the students to check their understanding - full answers plus advice must be given for these questions.

Distance education is already playing a role in astronomy, not only through the UK Open University, but also in France and elsewhere. However, the full potential of distance education in bringing astronomy, at all levels, to large numbers of people, has barely begun to be exploited. There is a golden opportunity here.

\title{
TEXTBOOKS AND ELECTRONIC MEDIA
}

Jay M. Pasachoff

Williams College-Hopkins Observatory, Williamstown, MA 01267, USA

E-mail: jay.m.pasachoff@williams.edu

1. Textbooks. Over the last few years, U.S. textbooks for the survey course have become increasingly sophisticated in the use of color diagrams and photographs and in the availability of supplemental material. This type of course is by far the largest exposure of astronomy to American students, with enrollments of over 200,000 per year. The increasing cost of preparing the books and auxiliary packages has led to many authors and publishing houses bringing out both higher and lower level books, with different lengths and different levels of mathematics.

Some 15 authors (or sets of co-authors) have brought out two dozen different textbooks with current copyrights (1993,1994, or 1995), including (with publishers in parentheses): Thomas T. Arny (Mosby), Eric Chaisson and Steve McMillan (Prentice-Hall), Sune Engelbrekston (Brown), William K. Hartmann (Wadsworth), William H. Jefferys and Robert R. Robbins (Wiley), James B. Kaler (HarperCollins), William J. Kaufmann III (Freeman), Karl F. Kuhn (West), Dinah L. Moché (Wiley), David Morrison and Sidney Wolff (Saunders; one book includes the late George Abell), Jay M. Pasachoff (Saunders), Michael A. Seeds (Wadsworth), Theodore P. Snow (West), and Michael Zeilik (Wiley). A table with lists and addresses is available on request.

Free supplements given to adopters of class-sized numbers of copies include special magazine or newspaper supplements, overhead transparencies of artwork and photographs, slide sets of artwork and photographs, videotapes, videodiscs, and 
computer programs, printed or computerized test banks of exam questions, and instructor's manuals including recommendations for audiovisual materials, laboratories, and answers to questions from the text.

2. Computer Planetaria. Computer programs are now available for personal computers that allow teachers and students to recreate the sky for any given time or place. In addition to simulating sky views, such astronomical phenomena as eclipses, conjunctions, occultations, daily motion, precession, and so on, can be simulated. Programs such as Voyager II for the Macintosh and Visible Universe and Dance of the Planets for IBM compatibles are examples of this genre. Similar programs for the IBM compatibles are Superstar, LodeStar Plus, The_Sky, PC-Sky, and StarGaze. The programs are available for less than $\$ 100$.

3. CD-ROM's. CD-ROM's are now available that include not only the above planetarium features but also hundreds of photographs, short movies, on-line astronomical dictionaries or encyclopedias, and other features. Further, the number of astronomical objects available is increased to even the 16 million stars in the Hubble Guide Star Catalogue, over 5000 asteroids, and so on. One of the most popular is RedShift, written in Russia by Russian scientists for an English company and marketed widely in the US to general audiences through computer stores and catalogues. I am writing some instructions and lessons to accompany it. Voyager II, Distant Suns and Guide are similar discs.

Other astronomical CD-ROM's include a disc about the 1991 total solar eclipse and a disk about the Murmurs from Earth set of images and recordings on a record being carried out of the solar system on the Voyager spacecraft.

Portable systems add a CD-ROM player weighing only about $1 \mathrm{~kg}$ to a laptop computer, and allow the demonstration of imaging and sky positions.

4. Videodiscs. The capabilities of laserdiscs include 54,000 frames on each side, so one can include thousands of still images plus approximately an hour of movies. The widely used videodisc on astronomy from 1984 was superseded in 1993 by a second edition called Beyond Earth. Images include NASA spacecraft movies, supercomputer simulations of black holes, Yohkoh $x$-ray solar images, and so on. For information, contact Optical Data Corp, 30 Technology Drive, P.O. Box 4919, Warren, NJ 07059, (fax: 908-668-1322). It is for the NTSC television standard.

I have consulted on both the above videodiscs and a videodisc for the Saunders astronomy texts: Saunders Videodisc Library \#1: The Solar System, released in 1994.

The American Association of Physics Teachers has a Physics Cinema Classics disc with many demonstrations.

5. NASA Space Educators' Handbook. A NASA scientist has put together a free series of Macintosh disks in Hypercard that include a dozen QuickTime very brief sound movies (a few seconds each) of space exploration and various written material. To receive them, send 10 high-density 3.5 " formatted Macintosh diskettes to Jerry Woodfill, NASA JSC (IA12), Houston, TX 77058.

6. Gems of Hubble. The Space Telescope Science Institute has prepared a series of "Electronic Picturebooks" for Macintosh computers, in which they use Hypercard to display a few dozen images and text on each subject. Subjects now available are Gems of Hubble (a set of highlights), Magellan Highlights of Venus, Images of Mars, 
The Planetary System, Apollo 11 at 25, Scientific Results from the Goddard High Resolution Spectrograph, The Impact Catastrophe that Ended the Mesozoic Era, and some other terrestrial subjects. They are available as computer disks or as a CDROM that includes them all. They can also be downloaded over the Internet. Get information from clallo@stsci.edu.

7. Astronomical Society of the Pacific. The Astronomical Society of the Pacific (390 Ashton Avenue, San Francisco, CA 94122, fax 415-337-5205, Internet: asp@stars.sfsu.edu) is a supplier of a wide variety of electronic media resources, including computer software, CD-ROM's and videodiscs.

8 . World Wide Web. The Internet has brought almost instant access to scientific data to many sites around the world. For example, the morning after observations were taken of the impact of Comet Shoemaker-Levy 9 on Jupiter, many observations from major observatories were posted on the Internet. Use of a graphics interface like Mosaic, and calling the Jet Propulsion Laboratory's "News Flashes" on www.jpl.nasa.gov, brought a choice of dozens of images via the World Wide Web.

\title{
RADIO ASTRONOMY SYSTEMS FOR UNDERGRADUATES
}

\author{
Aa. Sandqvist \\ Stockholm Observatory, S-133 36 Saltsjöbaden, Sweden \\ E-mail: sandqvis@astro.su.se \\ and \\ M. Hagström \\ Onsala Space Observatory, S-439 92 Onsala, Sweden
}

1. Introduction. In Sweden, there are four university departments of astronomy (Gothenburg, Lund, Stockholm and Uppsala) and one National Facility for Radio Astronomy, namely Onsala Space Observatory (OSO). Radio astronomy courses are offered only at OSO, which also hosts annual study visits by the country's advanced undergraduates in astronomy.

Teaching observational astronomy in Sweden has always been severely hampered by its high northern latitude and by its coastal climate. The light summer nights makes optical astronomy impossible during this season, and the large percentage of clouds and its unpredictability throughout the year almost annihilates organized class observational exercises.

As is well known, radio astronomical observations are possible both during the day and the night, as well as during cloudy conditions. It is therefore possible to plan schedules for undergraduate radio astronomy observing sessions without worrying about the weather or the time of day. "All" you need is the hardware and the software. We have therefore initiated a project for the construction of seven radio 